\title{
Characterising the Role of Small Airways in Severe Asthma using Low Frequency Forced Oscillations: A Combined Computational and Clinical Approach
}

${ }^{1 *}$ Brody H. Foy, ${ }^{2 *}$ Sushiladevi Natarajan, ${ }^{3}$ Arham Munawar, ${ }^{2}$ Marcia Soares, ${ }^{4}$ James Thorpe, ${ }^{4}$ John Owers-Bradley, ${ }^{2}$ Salman Siddiqui

${ }^{1}$ Computational Biology, Department of Computer Science, University of Oxford, Oxford, United Kingdom

${ }^{2}$ Institute for Lung Health, Department of Respiratory Sciences, University of Leicester, United Kingdom

${ }^{3}$ Birmingham Heartlands Hospital, Heart of England NHS Foundation Trust, Birmingham, United Kingdom

${ }^{4}$ School of Physics and Astronomy, University of Nottingham, Nottingham, United Kingdom.

*Contributed equally to the manuscript

Present address for correspondence:

Dr Brody Foy

Center for Systems Biology,

Simches Research Building

Massachusetts General Hospital

185 Cambridge Street, Boston, MA, 02114

e-mail: bfoy1@mgh.harvard.edu 


\section{DISCLOSURES}

\section{Ethics Approval and consent to participate}

This clinical study was approved by the National Research Ethics Committee - East Midlands Leicester (REC 08/H0406/189), and all subjects gave their written informed consent.

\section{Availability of data and materials}

The datasets generated during and/or analysed during the current study are available from the corresponding author on reasonable request

\section{Competing interests}

The authors declare that they have no competing interests.

\section{Funding}

This paper presents independent research funded by the National Institute for Health Research (NIHR). The views expressed are those of the authors and not necessarily those of the NHS, the NIHR or the Department of Health. Funding was received from the Airway Disease Predicting Outcomes through Patient Specific Computational Modelling (AirPROM) project (funded through an FP7 European Union grant).

\section{Author contributions:}

BHF designed the computational FOT model, performed all simulations, analysed the data, and co-wrote the manuscript. SN supported the clinical study design, recruitment in the clinical study, analysis of the FOT data in the clinical study and co-wrote the manuscript. AM designed the clinical study, recruited patients and conducted all of the FOT measurements in patients. MS supported patient recruitment in the clinical study. JT and JOB, developed the FOT device within the AirPROM-FP7 program, reviewed the manuscript and provided a scientific critique of the data, SS conceived and designed the study, coordinated the simulation work performed by BHF, analysed the data and co-wrote the manuscript with BHF and SN.

\section{Acknowledgements}

Not applicable.

Keywords: asthma, forced oscillation technique, deep inspiration, computational, small airways. 


\section{ABSTRACT}

\section{Background}

Within asthma, the small airways ( $\leq 2 \mathrm{~mm}$ in diameter) play an important role in pathophysiology. Using a combined clinical-computational approach, we sought to more precisely evaluate the contribution of the small airways to deep-breath induced airway dilation (in the absence of bronchial challenge), which may be impaired in severe asthma.

\section{Methods}

A patient-based computational model of the FOT was used to examine the sensitivity and specificity of FOT signals to small airways constriction at frequencies of $2 \& 8 \mathrm{~Hz}$. A clinical study of moderate to severe asthmatics $(n=24)$, and healthy volunteers $(n=10)$ was performed to evaluate correlations between baseline and post deep inspiration (following bronchodilator withhold and in the absence of prior bronchial challenge) forced oscillation technique (FOT) responses (at $2 \mathrm{~Hz}$ and $8 \mathrm{~Hz}$ ) and asthma treatment intensity, spirometry, airway hyper-responsiveness and airway inflammation.

\section{Results}

Computational modelling demonstrated that baseline resistance measures at $2 \mathrm{~Hz}$ are both sensitive and specific to anatomical narrowing in the small airways. Furthermore, small airways resistance was significantly increased in asthmatics compared to health. Despite these differences, there were no noticeable differences between asthmatics and healthy volunteers in resistive measures following deep inspiration (DI) and DI responses of small airways were amplified in the presence of spirometry defined airflow limitation.

\section{Conclusions}

These results suggest that the small airways demonstrate increased resistance in moderateto-severe asthma but dilate normally in response to deep inspirations in the absence of bronchial challenge. This suggests that effective targeting of the small airways is required to achieve functional improvements in moderate-severe asthmatic patients. 


\section{INTRODUCTION}

Asthma is a complex lung disease with a large global health burden, characterised by airway inflammation, remodelling, and increased smooth muscle activation ${ }^{1-4}$, all of which may result in heterogeneous small ( $\leq 2 \mathrm{~mm}$ diameter) airway obstruction, promoting ventilation heterogeneity and closure of both central and small ( $\leq 2 \mathrm{~mm}$ diameter) airways ${ }^{5}$, promoting ventilation heterogeneities ${ }^{6}$.

This heterogeneity may be particularly driven by inflammation of the small airways ${ }^{7,8}$. Recent studies of intact small airways ex vivo suggest that they remain sensitive to large pressure oscillations, with a bronchodilatory response [30]. However, studying the response of small airways to deep inspiration in vivo typically requires the application of low frequency forced oscillations $(<5 \mathrm{~Hz})$ during apnoea [12-14], due to the potential impact of higher order breathing harmonics [15].

In this study we were able to acquire reliable FOT data at $2 \mathrm{~Hz}$ without significant harmonic distortion during tidal breathing. We therefore sought to evaluate the impact of deep inspiration in vivo, on small airways using a combination of computational models as means of probing the anatomical sensitivity and specificity of FOT signals and a clinical study evaluating DI responses. DI responses were studied using FOT signals at both $2 \mathrm{~Hz}$ and $8 \mathrm{~Hz}$. We hypothesised that $2 \mathrm{~Hz}$ signal would be more sensitive to small airway narrowing and that the small airways would remain response to Dls even in severe asthma.

The clinical study was performed using deep inspiration (DI) in the absence of methacholine challenge, making it distinct from prior research in the literature ${ }^{9}$, and more representative of stable, exacerbation-free asthmatic airways. In addition DIs have a known bronchoprotective effect on asthmatic airways, following methacholine challenge ${ }^{10,11}$.

The primary focus of this study was to analyse FOT-derived resistance, however we also present results related to FOT-derived reactance in the online supplement.

\section{METHODS}

\section{Clinical recruitment}

34 adult subjects were recruited from Glenfield Hospital Leicester, and through local advertising and outpatient clinics. The group was comprised of 10 healthy volunteers, and 24 asthmatic subjects, as diagnosed by a secondary care clinician, with objective physiological evidence of asthma according to the British Thoracic Society (BTS)/SIGN guidelines ${ }^{12}$. All subjects were non-smokers at recruitment time, with a cumulative smoking history of $<10$ pack-years. All asthmatic subjects had not had an exacerbation in the 6 weeks prior to study and had been on stable asthma treatment. 
Asthma severity was classified according to the global initiative for asthma (GINA) treatment steps $^{2}$. All asthma subjects were at treatment steps 3-5 [ $n=8:$ GINA 3, $n=8:$ GINA 4, $n=8$ : GINA 5]. All but 3 subjects met ATS/ERS criteria for severe asthma ${ }^{2}$. For analysis, GINA 3 and 4 patients were pooled, due to similarities in therapeutics (both groups used moderate to high doses of inhaled steroids, with non-oral steroid based add on therapies, such as montelukast; whereas GINA 5 patients were all on oral systemic steroids).

This clinical study was approved by the National Research Ethics Committee - East Midlands Leicester (REC 08/H0406/189), and all subjects gave their written informed consent.

\section{Clinical visits}

The 24 asthmatic subjects attended up to three study visits, and underwent the following tests (all within \pm 14 days of each other):

- Visit 1: (i) Five-point Juniper Asthma control questionnaire (ACQ-5), (ii) skin-prick tests to common aeroallergens, (iii) spirometry (14), and (iv) sputum induction and processing for differential cell counts (16).

- Visit 2: A methacholine challenge using the tidal breathing method, if they had not had this performed in the past 2 years. This was used to confirm asthma diagnosis.

- Visit 3: FOT measurements during tidal breathing, according to a pre-specified DI protocol (Figure A1, online supplement).

All FOT measurements, spirometry and challenge tests were performed after withholding bronchodilators ( 4 hours for short acting agonists, and 12 hours for long acting $\beta 2$ agonists). Sputum differential cell counts were enumerated as eosinophilic airway inflammation is known to be associated with airflow limitation and ventilation heterogeneity ${ }^{13}$.

\section{FOT protocol and measurements}

Definitions of each FOT-derived clinical marker used within this study are given in the online supplement, Table A1. As primary measures we investigate total lung resistance (Rrs) and reactance $(\mathrm{Xrs})$ at $2 \mathrm{~Hz}(\mathrm{R} 2, \mathrm{X} 2)$, and $8 \mathrm{~Hz}(\mathrm{X} 2, \mathrm{X} 8)$, minimum resistance and reactance at the peak of a deep inspiration ( $\mathrm{Rmin}, \mathrm{Xmin}$ ), and the difference between minimum resistance/reactance and total lung resistance/reactance at $2 \mathrm{~Hz}(\Delta \mathrm{Rrs} 2 \mathrm{~Hz}, \Delta \mathrm{Xrs} 2 \mathrm{~Hz})$ and $8 \mathrm{~Hz}(\triangle \mathrm{Rrs} 8 \mathrm{~Hz}, \Delta \mathrm{Xrs} 8 \mathrm{~Hz})$. Alongside this, we consider the resistive and reactive difference from $2 \mathrm{~Hz}$ to $8 \mathrm{~Hz}$ (R2-R8, and $\mathrm{X} 2-\mathrm{X} 8$ ).

All measures of impedance of the respiratory system were performed using a custom built FOT device (see online supplement) designed in accordance with ERS task force guidelines ${ }^{15}$. This device was calibrated daily with standardised resistances in the respiratory impedance range. 
Full details of the FOT testing protocol are outlined in the online supplement. In brief, subjects sat in an upright position, and supported their cheeks around the mouthpiece, to reduce upper airway shunting. Each subject performed the test with $2 \mathrm{H}$ and $8 \mathrm{~Hz}$ single sine frequency sequences. Each sequence lasted for 150 seconds(s), consisting of a 60 s period of quiet tidal breathing, followed by three slow 30s deep inspirations (Dis), from functional residual capacity (FRC) up to total lung capacity (TLC). (see Figure $\mathbf{A} 1$ online supplement).

All FOT signals were measured during slow spontaneous tidal breathing, in contrast to standard protocol, for measurements to be made under apnoea ${ }^{15}$. We confirmed in a pilot study ( $\mathrm{n}-10$ subjects) that the both the $8 \mathrm{~Hz}$ and $2 \mathrm{~Hz}$ FOT signals generated highly repeatable within visit average resistances (Intra class correlation coefficient $>0.90$, see Table A2, online supplement) and that the $2 \mathrm{~Hz}$ signal was not significantly influenced by higher order breathing harmonics by evaluating Fourier transforms of the $2 \mathrm{~Hz}$ applied pressure signal during tidal breathing (see online supplement Figure A2).

Artefacts due to occasional leak, or glottic closure near peak DI were identified manually, and excluded. The baseline resistance pre-DI was calculated from the average of resistances during the first 50 seconds of pre-DI tidal breathing. Subsequently the minimum resistance at peak DI (Rmin) and change in resistance from baseline ( $\Delta \mathrm{Rrs})$ were calculated and averaged across six Dis from the two tidal breathing sequences.

\section{Computational simulated study}

Simulations of the FOT were performed using an electrical circuit analogous model, with full details presented in the online supplement. In short, a patient-based virtual conducting zone lung structure, was created through a combination of image segmentation, and algorithmic airway generation of a healthy patient CT scan, as reported in previous studies $^{16,17}$. This structure was comprised of approximately 71,000 branches, to an average depth of generation 16. Total lung impedance was calculated through parallel and series summation of individual branch impedances, which were approximated using the waveequation ${ }^{18,19}$. In prior studies, this model has been thoroughly investigated, and validated against experimental and clinical FOT measurements ${ }^{14,20-22}$.

Total lung impedance at $2 \mathrm{~Hz}$ and $8 \mathrm{~Hz}$ was calculated under various artificial constriction patterns, imposed on the virtual lung structure. For each simulation, all central airways $(2 \mathrm{~mm}<$ diameter $<10 \mathrm{~mm}$ ) or small airways (diameter $\leq 2 \mathrm{~mm}$ ) had radii reduced by a constriction factor $c(0-70 \%)$. This constriction factor was either applied homogeneously (using the same factor for each branch), or heterogeneously, by drawing each individual constriction from the normal distribution $\mathrm{N}(c, 0.2 c)$.

\section{Statistical analysis}


All parametric data was expressed as mean \pm standard deviation, and non-parametric data as median (interquartile range). Data was analysed using unpaired Student's t tests, or oneway analysis of variance (ANOVA), with Bonferroni correction for parametric data, and Mann-Whitney U test or Kruskal-Wallis test with Dunn's correction for non-parametric data, and Chi-squared or Fisher's exact tests for ratios. Stepwise linear regression was performed to determine the contributions of change in resistance ( $\Delta \mathrm{Rrs})$ at $2 \mathrm{~Hz}$ and $8 \mathrm{~Hz}$ to prebronchodilator FEV 1 FVC. Correlations between variables were calculated using Pearson's coefficient. A p-value of $<0.05$ was taken as the threshold for statistical significance.

\section{RESULTS}

\section{Computational analysis of airway sensitivity to $2 \mathrm{~Hz}$ and $8 \mathrm{~Hz}$ signals}

Results from the computational FOT simulations are presented in Figures 1-2. Figure 1 demonstrates that for all given levels of small airways constriction (10-70\%), resistance at $2 \mathrm{~Hz}$ (R2) was consistently more sensitive to small airway narrowing than resistance at $8 \mathrm{~Hz}$ (R8). In all cases the resistance increased non-linearly and was largest at constriction regimes $\geq 50 \%$. $R 2$ was also seen to increase at a faster rate than $R 8$, suggesting more sensitivity to small airways constriction. This behaviour was exhibited in both heterogeneous and homogeneous constriction schemes.

In Figure 2, we compare R2 and R8 under constriction of small and central airways. In both the heterogeneous and homogeneous scheme, small airways constriction is seen to produce larger R2 and R8 than central airways constriction. Equally, the relative increase of R2 comparative to $\mathrm{R} 8$ is much higher for small airways than central airways constriction Interestingly, all simulations in the heterogenous scheme appear to exhibit a small plateau as constriction rates become extremely large $(>65 \%)$. This is most likely due to the diminished role that heterogeneity can play as all airways become severely constricted.

This suggests that the $2 \mathrm{~Hz}$ signal is both sensitive and specific to constriction in the small airways. This data provided a rationale to make inferences of small airway physiology in $2 \mathrm{~Hz}$ data from the clinical study.

Reactance simulation results are presented in the online supplement Figures A3-A4.

Central and small airway impedance in asthma at baseline and following deep inspiration The clinical FOT, and spirometry measurements, and demographic data for both asthmatic and healthy subjects is shown in Tables 1-2. The healthy and asthmatic subjects were well matched for age, sex, and body mass index. Both R2 and R8 were shown to be significantly greater in asthmatics than the healthy controls (Figure 3) [R2: $0.66(0.31)$ vs $0.44(0.12)$ kPa.s.L $L^{-1}, p=0.04$; R8: 0.45 (0.19) vs 0.31 (0.10) kPa.s.L $\left.L^{-1}, p=0.04\right]$. 
In contrast to the baseline resistance, there was no apparent difference in minimum resistance at peak DI (Rmin) at either $2 \mathrm{~Hz}$ or $8 \mathrm{~Hz}$, between asthmatic subjects and healthy controls. Additionally, there was a no significant difference in mean resistive difference ( $\Delta \mathrm{Rrs}$, the difference between baseline resistance and Rmin) between asthmatics and healthy controls, at either $2 \mathrm{~Hz}$ or $8 \mathrm{~Hz}$.

No significant correlation was observed between Rmin and methacholine $\mathrm{PC}_{20}$ at either $2 \mathrm{~Hz}$ $(r=-0.10, p=0.68)$ or $8 \mathrm{~Hz}(r=-0.22, p=0.68)$. There was however, a modest and statistically significant correlation between $\mathrm{Rmin}$ at $8 \mathrm{~Hz}$, and percentage sputum eosinophils $(r=0.4318, p=0.04)$, though this was not observed with the $2 \mathrm{~Hz}$ signal.

The resistive difference following $\mathrm{DI}(\triangle \mathrm{Rrs})$ correlated negatively with pre-bronchodilator $\mathrm{FEV}_{1} / \mathrm{FVC}$, at both $2 \mathrm{~Hz}(r=-0.51, p=0.01)$ and $8 \mathrm{~Hz}(r=-0.48, p=0.02)$. An independent association between pre-bronchodilator $F E V_{1} / F V C$ and $\Delta R r s$ at $2 \mathrm{~Hz}$ was identified $\left(R^{2}=0.24\right.$, $p=0.01)$.

Clinical reactance results are given in the online supplement, Tables A3-A4, and Figure A5.

\section{DISCUSSION}

Within this study a combination of computational modelling and clinical data analysis was used to study the role of the small airways using low frequency FOT in response to deep inspiration in patient with severe asthma. The computational analyses confirmed that the $2 \mathrm{~Hz}$ FOT signal has greater sensitivity than the $8 \mathrm{~Hz}$ signal to anatomical narrowing in the small airways than central airways in both the presence and absence of constriction heterogeneity (Figures 1-2). The clinical study confirmed that $2 \mathrm{~Hz}$ FOT measurements could be performed repeatably and reliably and that despite elevated baseline small airway resistance, the small airways dilate normally in response to a deep inspiration in asthma in the non-challenged state.

The key observation here, that small airways retain their ability to respond to DI in severe asthma would suggest that the airways remain amenable to intervention with targeted strategies that provide further pharmacological bronchoprotection. This hypothesis would need to be tested in future small airway targeted intervention studies.

\section{Interpretation of the key findings}

The use of a clinically-validated computational airway model ${ }^{14,23}$, simulated upon a patientbased virtual lung structure allowed us to explore structure-function relationships in novel ways, providing more clarity to clinical interpretation of the $2 \mathrm{~Hz}$ and $8 \mathrm{~Hz}$ FOT signals in this study. 
Disease in the small airways has been described in multiple imaging and immunopathological studies in asthma ${ }^{24-26}$. Our observations of elevated baseline resistance at $2 \mathrm{~Hz}$ in combination with the anatomical sensitivity demonstrated by computational modelling further add support to this concept. Furthermore, the application of a tidal breathing $2 \mathrm{~Hz}$ technique to study small airway physiology provide a future opportunity for translational studies of small airways disease using FOT in larger clinical populations.

In contrast to baseline observations, the minimum resistance post-DI (Rmin), and mean resistive difference from $\mathrm{DI}$ to baseline ( $\Delta \mathrm{Rrs}$ ) at $2 \mathrm{~Hz}$ did not differ significantly in the presence of asthma. This suggests that while asthmatic airways exhibit larger degrees of narrowing at baseline, their response to deep inspiration is not abnormal. This is congruent with more recent work in the literature, which suggests that in asthmatics, the small airways remain compliant to large pressure oscillations ${ }^{27}$.

We also note that the $\Delta \mathrm{Rrs}$ signal at $2 \mathrm{~Hz}$ demonstrated an inverse association with prebronchodilator $F E V_{1} / F V C$, suggesting that expiratory flow limiting mechanisms may also be the same mechanisms responsible for the magnitude of DI response in the small airways.

Joint interpretation of the computational and clinical results suggests that the $2 \mathrm{~Hz}$ signal shows greater sensitivity to anatomical narrowing in the small airway tree, and that unlike at $8 \mathrm{~Hz}$, the airways which contribute most heavily to the $2 \mathrm{~Hz}$ signal are quite responsive to deep inspiration.

A recent study demonstrated using HP-gas imaging that ventilation defects were more likely to persists after bronchodilators if patients had a sputum eosinophilia ${ }^{13}$. Our observations support this hypothesis showing that that sputum eosinophilia correlated positively with the Rmin (the minimum resistance that could be achieved after a DI) at $8 \mathrm{~Hz}$ (reflective of airway disease as opposed to parenchymal disease). Sputum eosinophils may modify bronchial tone, as they reflect mucosal eosinophils which promote airway smooth muscle contractional, through paracrine signalling with the airway smooth muscle via mediator release (e.g. cysteinyl leukotrienes). It is therefore plausible that bronchoprotection in severe asthma could be better achieved through more effective targeting of eosinophilic airway inflammation, for example using eosinophil lowering biologics targeting IL-5.

\section{Comparisons to R5-R20}

Within the literature the use of R5-R20 as a measure of small airways dysfunction has gained traction ${ }^{14}$, which in part has motivated the consideration of R2-R8. Given R8 is likely to be close to resonant frequency, it may mostly reflect airway resistance contributions, while R2 is more likely to be influenced by parenchymal dynamics and lung reactance. R5R20 takes measures of resistance from (typically) above and below resonant frequency, meaning there are influences of negative reactance on the $5 \mathrm{~Hz}$ signal, and positive 
reactance on the $20 \mathrm{~Hz}$ signal. $\mathrm{R} 2-\mathrm{R} 8$ instead takes a measure with negative reactance influences, and a measure with minimal reactance influences, isolating the reactance effects more. We believe that the results within this study illustrate that the $2 \mathrm{~Hz}$ signal can be collected without induced apnoea, allowing for wider investigations of R2-R8 as a resistive difference measure which may be complementary to the more widely used R5-R20.

\section{Modelling deep inspiration without methacholine challenge}

Unlike most similar studies within the literature, clinical data collection was performed in a non-challenge model, with bronchodilators withheld to allow appropriate pre-testing washout. This choice was actively made to analyse the response of small airways in a physiological state that mimics stable asthma.

In stable asthma several potentially therapeutically targetable factors promote narrowing of small airways including mucous plugging ${ }^{28}$, airway surfactant dysfunction leading to airways closure $^{29}$, airway smooth muscle activation ${ }^{30}$, altered alveolar parenchymal attachments ${ }^{31}$ and remodelling of the airway walls ${ }^{25}$. All these factors would increase small airways resistance, however the demonstration of bronchoprotection would point towards factors that can be reversed over a short time course such as airway smooth muscle activation and airways closure.

The prior bronchial challenge DI studies in the literature are much more in keeping with an exacerbation state. Our study therefore, adds insight into the potential for therapeutic intervention in stable asthma targeted at bronchoprotection of the small airways. This may explain why the results we have illustrated appear to contradict prior findings ${ }^{9}$, as these findings are post bronchial challenge, and thus more likely to represent an exacerbation state, than the lung state we have studied.

Schweitzer et al. ${ }^{32}$ published one of the few pre-bronchodilator, non-challenged, tidal breathing studies and demonstrated negligible improvement in both inspiratory and expiratory resistance at $12 \mathrm{~Hz}$ (using a loudspeaker around the head) following a DI in a group of asthmatic children with varying levels of spirometric airflow obstruction. These results are in keeping with our findings at $8 \mathrm{~Hz}$. Furthermore, the $\mathrm{DI}$ response was smaller in patients with greater airflow obstruction. We identified greater responses to DI at both 2 and $8 \mathrm{~Hz}$ in the presence of increased airflow limitation, several factors may account for the discrepancy in results.

Firstly, we evaluated pressure oscillations applied directly to the mouth rather than via a head unit, our study focussed on low frequencies $(2 \& 8 \mathrm{~Hz})$ typically below resonant frequency where parenchymal dynamics would be expected to contribute to the frequency response and our study was limited to adults with severe asthma that did not have severe airflow limitation. It is possible, that in the presence of severe background airflow limitation 
paradoxical responses are observed in the small airways in response to $\mathrm{DI}$, however this was not the focus of the current study.

\section{A broader role for anatomically driven computational models}

Alongside the clinical findings, these results also add evidence to the role that combined computational-clinical analysis can play in understanding pulmonary function (and associated clinical testing). In a variety of recent studies ${ }^{14,20,33}$, we have shown how anatomically-based computational modelling can lead to unique and clinically relevant insights into pulmonary function tests. The use of CT-based virtual lung geometries is still a relatively new endeavour, but one that shows great promise, particularly when combined with clinical data analysis. In particular, within this study we have shown that small airways remain responsive to $\mathrm{DI}$, validating resistance at $2 \mathrm{~Hz}$ as a sensitive and specific index of small airways disease.

\section{Limitations}

Results should be interpreted with reference to the limitations of the computational modelling protocol. Due to computational and imaging constraints, the model makes a series of simplifying assumptions, including the incorporation of homogeneous peripheral elastance (constant-phase model), and the assumption of static airway radii, each of which may introduce inaccuracy in the simulations. To account for this, in prior studies ${ }^{14,21}$ the ability of the model to accurately simulate resistance (both in a clinical context, and against experimental values from a 3D printed lung structure) was validated. However, this validation was performed in a higher frequency range $(5-25 \mathrm{~Hz})$ and was not performed for reactance measures. For this reason, reactance measures have been included in supplemental material only, to reflect the lower degree of confidence in simulation outputs. In prior work we have illustrated how newer image analysis techniques may allow for higher quality structural information ${ }^{34}$, which could be used to better parameterise respiratory zone contributions, and small airway diameters. In future work we hope to apply these techniques to allow for a more robust, and clinically validated reactance model.

Considering the clinical data, as discussed earlier in the manuscript, all measurements were taken during spontaneous tidal breathing, in a non-challenged, pre-bronchodilator state. This setup was chosen to more accurately replicate stable, non-exacerbated asthmatic airways. In particular, the choice to measure during spontaneous breathing, is contrary to typical procedure ${ }^{15}$, due to the potential influence of spontaneous breathing frequencies on the low-frequency response. However, as illustrated in the supplemental material, FOT measurements at $2 \mathrm{~Hz}$ showed strong within-visit repeatability and negligible interference from higher order breathing harmonics when the frequency contents of the $2 \mathrm{~Hz}$ pressure oscillation was evaluated at the mouth using FFT. These differences in setup may partly explain discrepancies between the results from our study, and post-challenge studies ${ }^{9}$. 
Within future research we hope to more robustly analyse FOT signals at low frequency under a wider variety of clinical experimental conditions.

\section{CONCLUSIONS}

Within this study, we have used a combinational of computational modelling and clinical evaluation to study the role of the small airways in severe asthma. We demonstrate that the small airways dilate normally in response to deep inspiration with amplified response in the presence of spirometry defined airflow obstruction. We hypothesise that the small airways remain sensitive to bronchoprotective therapies in severe asthma.

\section{REFERENCES}

1. Carroll N, Elliot J, Morton A, James A. The Structure of Large and Small Airways in Nonfatal and Fatal Asthma. Am Rev Respir Dis. 2013. doi:10.1164/ajrccm/147.2.405

2. Chung KF, Wenzel SE, Brozek JL, et al. International ERS/ATS guidelines on definition, evaluation and treatment of severe asthma. Eur Respir J. 2014. doi:10.1183/09031936.00202013

3. Stephens NL, Cheng ZQ, Fust A. Sensitized airway smooth muscle plasticity and hyperreactivity: a review. Can J Physiol Pharmacol. 2007.

4. Ward C, Johns DP, Bish R, et al. Reduced airway distensibility, fixed airflow limitation, and airway wall remodeling in asthma. Am J Respir Crit Care Med. 2001. doi:10.1164/ajrccm.164.9.2102039

5. Ranga V, Kleinerman J. Structure and function of small airways in health and disease. Arch Pathol Lab Med. 1978.

6. Venegas JG, Winkler T, Musch G, et al. Self-organized patchiness in asthma as a prelude to catastrophic shifts. Nature. 2005. doi:10.1038/nature03490

7. Wagner EM, Liu MC, Weinmann GG, Permutt S, Bleecker ER. Peripheral Lung Resistance in Normal and Asthmatic Subjects. Am Rev Respir Dis. 2011. doi:10.1164/ajrccm/141.3.584

8. Yanai M, Sekizawa K, Ohrui T, Sasaki H, Takishima T. Site of airway obstruction in pulmonary disease: direct measurement of intrabronchial pressure. J Appl Physiol. 2017. doi:10.1152/jappl.1992.72.3.1016

9. Slats AM, Janssen K, Van Schadewijk A, et al. Bronchial inflammation and airway responses to deep inspiration in asthma and chronic obstructive pulmonary disease. Am J Respir Crit Care Med. 2007. doi:10.1164/rccm.200612-18140C

10. Jensen A, Atileh H, Suki B, Ingenito EP, Lutchen KR. Selected Contribution: Airway caliber in healthy and asthmatic subjects: effects of bronchial challenge and deep inspirations. J Appl Physiol. 2017. doi:10.1152/jappl.2001.91.1.506

11. Salome CM, Thorpe CW, Diba C, Brown NJ, Berend N, King GG. Airway re-narrowing following deep inspiration in asthmatic and nonasthmatic subjects. Eur Respir J. 2003. doi:10.1183/09031936.03.00117502

12. British Thoracic Society/Scottish Intercollegiate Guidelines Network. British Guideline on the Management of Asthma. https://www.brit-thoracic.org.uk/guidelines-and- 
quality-standards/asthma-guideline/. Published 2014.

13. Svenningsen S, Eddy RL, Lim HF, Cox PG, Nair P, Parraga G. Sputum eosinophilia and magnetic resonance imaging ventilation heterogeneity in severe asthma. Am J Respir Crit Care Med. 2018. doi:10.1164/rccm.201709-19480C

14. Foy BH, Soares M, Bordas R, et al. Lung Computational Models and the Role of the Small Airways in Asthma. Am J Respir Crit Care Med. 2019. doi:10.1164/rccm.201812$23220 \mathrm{C}$

15. Oostveen $\mathrm{E}$, MacLeod $\mathrm{D}$, Lorino $\mathrm{H}$, et al. The forced oscillation technique in clinical practice: Methodology, recommendations and future developments. Eur Respir J. 2003. doi:10.1183/09031936.03.00089403

16. Bordas R, Lefevre C, Veeckmans B, et al. Development and analysis of patient-based complete conducting airways models. PLoS One. 2015. doi:10.1371/journal.pone.0144105

17. Tawhai MH. CT-based geometry analysis and finite element models of the human and ovine bronchial tree. J Appl Physiol. 2004. doi:10.1152/japplphysiol.00520.2004

18. Benade AH. On the Propagation of Sound Waves in a Cylindrical Conduit. J Acoust Soc Am. 1968. doi:10.1121/1.1911130

19. Thurston G. Periodic fluid flow through circular tubes. J Acoust Soc Am. 1952;24(6):653-656.

20. Foy BH, Kay D. A computational comparison of the multiple-breath washout and forced oscillation technique as markers of bronchoconstriction. Respir Physiol Neurobiol. 2017. doi:10.1016/j.resp.2017.02.016

21. Soares M, Owers-Bradley J, Foy B, Kay D, Siddiqui S. The evaluation of frequency dependence of resistance using a patient-specific 3D physical model and a computational model. In: ; 2017. doi:10.1183/1393003.congress-2017.pa2476

22. Foy BH, Bell AJ, Siddiqui S, Kay D. Low frequency lung resistance is a global bronchoconstriction detection measure, but is still sensitive to small airways disease. American Thoracic Society Congress. 2018.

23. Foy BH, Kay D. A computationally tractable scheme for simulation of the human pulmonary system. J Comput Phys. 2019;388:371-393. doi:10.1016/j.jcp.2019.03.014

24. Lipworth B, Manoharan A, Anderson W. Unlocking the quiet zone: The small airway asthma phenotype. Lancet Respir Med. 2014. doi:10.1016/S2213-2600(14)70103-1

25. Kuwano K, Bosken CH, Pare PD, Bai TR, Wiggs BR, Hogg JC. Small airways dimensions in asthma and in chronic obstructive pulmonary disease. Am Rev Respir Dis. 1993. doi:10.1164/ajrccm/148.5.1220

26. Bittar HET, Doberer D, Mehrad M, et al. Histologic findings of severe/therapyresistant asthma from video-assisted thoracoscopic surgery biopsies. Am J Surg Pathol. 2017. doi:10.1097/pas.0000000000000777

27. Harvey BC, Parameswaran H, Lutchen KR. Can breathing-like pressure oscillations reverse or prevent narrowing of small intact airways? J Appl Physiol. 2015. doi:10.1152/japplphysiol.01100.2014

28. Dunican EM, Elicker BM, Gierada DS, et al. Mucus plugs in patients with asthma linked to eosinophilia and airflow obstruction. J Clin Invest. 2018. doi:10.1172/JCl95693

29. Yager D, Kamm RD, Drazen JM. Airway wall liquid. Sources and role as an amplifier of bronchoconstriction. Chest. 1995.

30. Pera T, Penn RB. Bronchoprotection and bronchorelaxation in asthma: New targets, and new ways to target the old ones. Pharmacol Ther. 2016. 
doi:10.1016/j.pharmthera.2016.04.002

31. Mauad T, Silva LFF, Santos MA, et al. Abnormal alveolar attachments with decreased elastic fiber content in distal lung in fatal asthma. Am J Respir Crit Care Med. 2004. doi:10.1164/rccm.200403-3050C

32. Schweitzer C, Moreau-Colson C, Marchal F. Respiratory impedance response to a deep inhalation in asthmatic children with spontaneous airway obstruction. Eur Respir J. 2002. doi:10.1183/09031936.02.00992001

33. Foy BH, Kay D, Siddiqui S, Paiva M, Verbanck S. Increased ventilation heterogeneity in asthma can be attributed to proximal bronchioles. Eur Respir J. 2019; In Press.

34. Bell AJ, Foy BH, Richardson $M$, et al. Functional CT imaging for identification of the spatial determinants of small-airways disease in adults with asthma. J Allergy Clin Immunol. 2019. doi:10.1016/j.jaci.2019.01.014 
TABLES AND FIGURE LEGENDS

Table 1: Demographics, clinical characteristics and resistance measurements between asthmatics and healthy controls

\begin{tabular}{|c|c|c|c|}
\hline & $\begin{array}{l}\text { Healthy } \\
(n=10)\end{array}$ & $\begin{array}{l}\text { Asthmatic } \\
(n=24)\end{array}$ & $p$-value \\
\hline Age (years) & $52(16.1)$ & $57.38(13.3)$ & 0.34 \\
\hline Sex $(\% \text { male })^{\S}$ & 20 & 33.3 & 0.37 \\
\hline $\mathrm{BMI}\left(\mathrm{Kg} / \mathrm{m}^{2}\right)$ & $26.4(5.2)$ & $28.3(5.5)$ & 0.37 \\
\hline Smoking history (pack years) & $1.0(0.0-3.5)$ & $0.0(0.0-3.5)$ & 0.83 \\
\hline Duration of asthma (years) & $\mathrm{n} / \mathrm{a}$ & $28.79(18.4)$ & - \\
\hline Atopy (\% yes) $)^{\S, *}$ & 33.3 & $\begin{array}{l}82.6 \\
{[n=23]}\end{array}$ & 0.006 \\
\hline ICS [BDP equivalent $(\mathrm{mcg})]^{\uparrow}$ & $\mathrm{n} / \mathrm{a}$ & $\begin{array}{l}800 \\
(500-1000)\end{array}$ & $\mathrm{n} / \mathrm{a}$ \\
\hline Oral prednisolone $(\mathrm{mg})^{n}$ & $\mathrm{n} / \mathrm{a}$ & $0.0(0.0-5.0)$ & $\mathrm{n} / \mathrm{a}$ \\
\hline ACQ-5 score & $\mathrm{n} / \mathrm{a}$ & $\begin{array}{l}1.57(1.14-2.57) \\
{[n=19]}\end{array}$ & $\mathrm{n} / \mathrm{a}$ \\
\hline Exacerbations in previous year & $\mathrm{n} / \mathrm{a}$ & $1.0(0.0-2.0)$ & $\mathrm{n} / \mathrm{a}$ \\
\hline Sputum neutrophils (\%) & $\begin{array}{l}71.68(30.3) \\
{[n=7]}\end{array}$ & $64.52(22.34)$ & 0.50 \\
\hline Sputum eosinophils (\%) & $\begin{array}{l}0.25 \\
(0.25-2.5) \\
{[n=7]}\end{array}$ & $\begin{array}{l}1.75 \\
(0.50-7.25)\end{array}$ & 0.23 \\
\hline Pre BD FEV 1 (\% Pred)* & $\begin{array}{l}105.8(21.4) \\
{[n=8]}\end{array}$ & $85.78(24.5)$ & 0.05 \\
\hline Pre BD FEV $1 / F V C$ (\% Pred) & $\begin{array}{l}77.88(5.4) \\
{[n=8]}\end{array}$ & $71.97(9.38)$ & 0.11 \\
\hline Post BD FEV $1 /$ FVC (\% Pred) & $\begin{array}{l}80.9(5.4) \\
{[n=8]}\end{array}$ & $74.64(10.73)$ & 0.13 \\
\hline BD reversibility ${ }^{\uparrow, *}$ & $\begin{array}{l}-0.325(-2.04-3.77) \\
{[n=8]}\end{array}$ & $\begin{array}{l}5.6 \\
(3.33-8.82)\end{array}$ & 0.01 \\
\hline PC20 methacholine $(\mathrm{mg} / \mathrm{ml})^{\mathrm{n}, *}$ & $16.0(16.0-16.0)$ & $\begin{array}{l}6.35(0.94-16.0) \\
{[n=18]}\end{array}$ & 0.003 \\
\hline $\mathrm{R} 8 \mathrm{~Hz}\left(\text { Kpa.s. } \mathrm{L}^{-1}\right)^{*}$ & $0.31(0.10)$ & $0.45(0.19)$ & 0.04 \\
\hline $\mathrm{R} 2 \mathrm{~Hz}\left(\text { Kpa.s.L }{ }^{-1}\right)^{*}$ & $0.44(0.12)$ & $0.66(0.31)$ & 0.04 \\
\hline R2-R8 (Kpa.s.L-1) & $0.13(0.04)$ & $0.18(0.18)$ & 0.41 \\
\hline Rmin $8 \mathrm{~Hz}\left(\right.$ Kpa.s. $\left.\mathrm{L}^{-1}\right)$ & $0.19(0.06)$ & $0.23(0.12)$ & 0.27 \\
\hline $\mathrm{Rmin} 2 \mathrm{~Hz}\left(\right.$ Kpa.s. $\left.\mathrm{L}^{-1}\right)$ & $0.27(0.06)$ & $0.36(0.21)$ & 0.19 \\
\hline$\Delta \mathrm{Rrs} 2 \mathrm{~Hz}\left(\right.$ Kpa.s.L $\left.\mathrm{L}^{-1}\right)$ & $0.17(0.09)$ & $0.27(0.31)$ & 0.34 \\
\hline$\Delta \mathrm{Rrs} 8 \mathrm{~Hz}\left(\right.$ Kpa.s. $\left.\mathrm{L}^{-1}\right)$ & $0.13(0.06)$ & $0.22(0.17)$ & 0.10 \\
\hline Rrs decay $2 \mathrm{~Hz}$ (seconds) & $4.92(1.70)$ & $4.00(1.76)$ & 0.14 \\
\hline Rrs decay $8 \mathrm{~Hz}$ (seconds) & $4.46(2.68)$ & $4.88(2.27)$ & 0.64 \\
\hline
\end{tabular}

$\mathrm{BMI}=$ Body mass index; ICS = Inhaled corticosteroids; BDP = Beclomethasone dipropionate; ACQ -5 = 5 point asthma control questionnaire; $\mathrm{BD}=$ Bronchodilator; $\mathrm{FEV}_{1}=$ Forced expiratory volume in one second; $\mathrm{FVC}=$ Forced vital capacity; GINA = Global initiative for asthma; R2 = Mean baseline resistance at 2Hz; R8 = Mean baseline resistance at $8 \mathrm{~Hz} ; \mathrm{Rrs}=$ Resistance of respiratory system; Rmin = minimum resistance achieved at maximal deep inspiration. Data expressed as mean [Standard deviation (SD)] for parametric data, "median [Interquartile range (IQR)] for non-parametric data. Groups compared using student t test for parametric data or Mann whitney test for non-parametric data. ${ }^{\S}$ Fishers exact test for proportions. Significant difference between healthy controls and asthmatics denoted $*(p<0.05)$. 
Table 2: Demographics, clinical characteristics and resistance measurements by GINA treatment steps

\begin{tabular}{|c|c|c|c|c|}
\hline & $\begin{array}{l}\text { Healthy } \\
(n=10)\end{array}$ & $\begin{array}{l}\text { GINA 3/4 } \\
(n=16(8 / 8))\end{array}$ & $\begin{array}{l}\text { GINA } 5 \\
(n=8)\end{array}$ & $p$-value \\
\hline Age (Years) & $52(16.1)$ & $59.94(11.9)$ & $52.25(15.2)$ & 0.29 \\
\hline Sex $(\% \text { male })^{\S}$ & 20 & 37.5 & 37.5 & 0.61 \\
\hline $\mathrm{BMI}\left(\mathrm{Kg} / \mathrm{m}^{2}\right)$ & $26.4(5.2)$ & $28.3(5.94)$ & $28.1(4.88)$ & 0.67 \\
\hline $\begin{array}{l}\text { Smoking history } \\
\text { (Pack years)" }\end{array}$ & $1.0(0.0-3.5)$ & $0.0(0.0-3.4)$ & $0.50(0.0-6.5)$ & 0.77 \\
\hline Duration of asthma (years) & $\mathrm{n} / \mathrm{a}$ & $25.75(18.7)$ & $34.88(17.3)$ & 0.26 \\
\hline Atopy $(\% \text { yes) })^{\S}$ & 33.3 & 81.3 & $\begin{array}{l}85.7 \\
{[n=7]}\end{array}$ & 0.02 \\
\hline ICS [BDP equivalent $(\mathrm{mcg})]^{\pi}$ & $\mathrm{n} / \mathrm{a}$ & $\begin{array}{l}800 \\
(400-800)\end{array}$ & $\begin{array}{l}1000 \\
(850-1450)\end{array}$ & 0.007 \\
\hline Oral prednisolone (mg) & $\mathrm{n} / \mathrm{a}$ & $\mathrm{n} / \mathrm{a}$ & $\begin{array}{l}7.5 \\
(5.0-10) \\
\end{array}$ & $\mathrm{n} / \mathrm{a}$ \\
\hline ACQ-5 score & $\mathrm{n} / \mathrm{a}$ & $1.62(0.86)[n=12]$ & $1.88(0.89)$ & 0.53 \\
\hline Exacerbations in previous year" & $\mathrm{n} / \mathrm{a}$ & $\begin{array}{l}1.0 \\
(0.0-2.0) \\
\end{array}$ & $\begin{array}{l}1.0 \\
(0.0-2.0) \\
\end{array}$ & 0.65 \\
\hline Sputum neutrophils (\%) & $\begin{array}{l}71.68(30.3) \\
{[n=7]}\end{array}$ & $\begin{array}{l}63.5(23.1) \\
{[n=15]}\end{array}$ & $66.5(22.3)$ & 0.77 \\
\hline Sputum eosinophils (\%)" & $\begin{array}{l}0.25 \\
(0.25-2.5) \\
{[n=7]}\end{array}$ & $\begin{array}{l}1.75 \\
(0.50-7.25) \\
{[n=15]} \\
\end{array}$ & $\begin{array}{l}2.50 \\
(0.25-15.06)\end{array}$ & 0.37 \\
\hline Pre BD FEV 1 (\% pred) & $\begin{array}{l}105.8(21.4) \\
{[n=8]}\end{array}$ & $92.1(23.4)$ & $71.4(22.1)^{*}$ & 0.02 \\
\hline Pre BD FEV $1 / F V C$ (\% pred) & $\begin{array}{l}77.88(5.4) \\
{[n=8]}\end{array}$ & $73.7(8.85)$ & $68.0(10.0)$ & 0.09 \\
\hline Post BD FEV $1 / F V C$ (\% pred) & $\begin{array}{l}80.9(5.4) \\
{[n=8]}\end{array}$ & $76.4(10.0)$ & $71.9(12.7)$ & 0.21 \\
\hline $\mathrm{BD}$ reversibility $(\%)^{\natural}$ & $\begin{array}{l}-0.325 \\
(-2.04-(-3.77)) \\
{[n=8]}\end{array}$ & $\begin{array}{l}5.17 \\
(2.43-8.43)\end{array}$ & $\begin{array}{l}5.70 \\
(5.50-34.87)^{*}\end{array}$ & 0.02 \\
\hline $\mathrm{PC}_{20}$ Methacholine $(\mathrm{mg} / \mathrm{ml})^{\pi}$ & $16.0(16.0-16.0)$ & $\begin{array}{l}8.0 \\
(1.83-16.0)^{*} \\
{[n=13]}\end{array}$ & $\begin{array}{l}1.0 \\
(0.39-14.6)^{*} \\
{[n=5]}\end{array}$ & 0.008 \\
\hline R8 Hz (Kpa.s.L L $^{-1}$ ) & $0.31(0.10)$ & $0.41(0.17)$ & $0.54(0.22)^{*}$ & 0.03 \\
\hline R2 Hz (Kpa.s.L L $^{-1}$ ) & $0.44(0.12)$ & $0.62(0.29)$ & $0.72(0.34)$ & 0.09 \\
\hline R2-R8 (Kpa.s. L-11) & $0.13(0.04)$ & $0.17(0.20)$ & $0.18(0.15)$ & 0.71 \\
\hline Rmin $8 \mathrm{~Hz}\left(\right.$ Kpa.s. L $\left.^{-1}\right)$ & $0.19(0.06)$ & $0.19(0.08)$ & $0.32(0.15)^{*}$ & 0.01 \\
\hline Rmin $2 \mathrm{~Hz}\left(\right.$ Kpa.s. L $\left.^{-1}\right)$ & $0.27(0.06)$ & $0.37(0.26)$ & $0.35(0.08)$ & 0.40 \\
\hline$\Delta \operatorname{Rrs} 2 \mathrm{~Hz}\left(\right.$ Kpa.s.L $\left.{ }^{-1}\right)$ & $0.17(0.09)$ & $0.21(0.32)$ & $0.38(0.29)$ & 0.23 \\
\hline$\Delta \operatorname{Rrs} 8 \mathrm{~Hz}\left(\right.$ Kpa.s. L $\left.{ }^{-1}\right)$ & $0.13(0.06)$ & $0.22(0.11)$ & $0.22(0.27)$ & 0.26 \\
\hline Rrs decay $2 \mathrm{~Hz}$ (seconds) & $4.92(1.70)$ & $3.70(2.02)$ & 4.35 (1.09) & 0.23 \\
\hline Rrs decay $8 \mathrm{~Hz}$ (seconds) & $4.46(2.68)$ & $4.82(2.22)$ & $5.01(2.52)$ & 0.88 \\
\hline
\end{tabular}

GINA = Global initiative for asthma; BMI = Body mass index; ICS = Inhaled corticosteroids; BDP =

Beclomethasone dipropionate; $\mathrm{ACQ}-5=5$ point asthma control questionnaire; $\mathrm{BD}=$ Bronchodilator; $\mathrm{FEV}_{1}=$ Forced expiratory volume in one second; $\mathrm{FVC}=$ Forced vital capacity; $\mathrm{PC}_{20}=$ Provocation concentration causing $20 \%$ fall in $\mathrm{FEV}_{1} ; \mathrm{R} 2=$ Mean baseline resistance at $2 \mathrm{~Hz} ; \mathrm{R} 8=$ Mean baseline resistance at $8 \mathrm{~Hz} ; \mathrm{Rmin}=$ minimum resistance achieved at maximal deep inspiration; Rrs = Resistance of respiratory system. Data expressed as mean [Standard deviation (SD)] for parametric data, "median [Interquartile range (IQR)] for non-parametric data. Groups compared using one-way Analysis of variance (ANOVA) for parametric data with Bonferonni correction for multiple comparisons or Kruskal-Wallis test with Dunn correction for non-parametric data. ${ }^{\S} \mathrm{Chi}$ squared test for proportions. Significant difference between healthy controls and GINA 5 asthma denoted $*(p<0.05)$. 


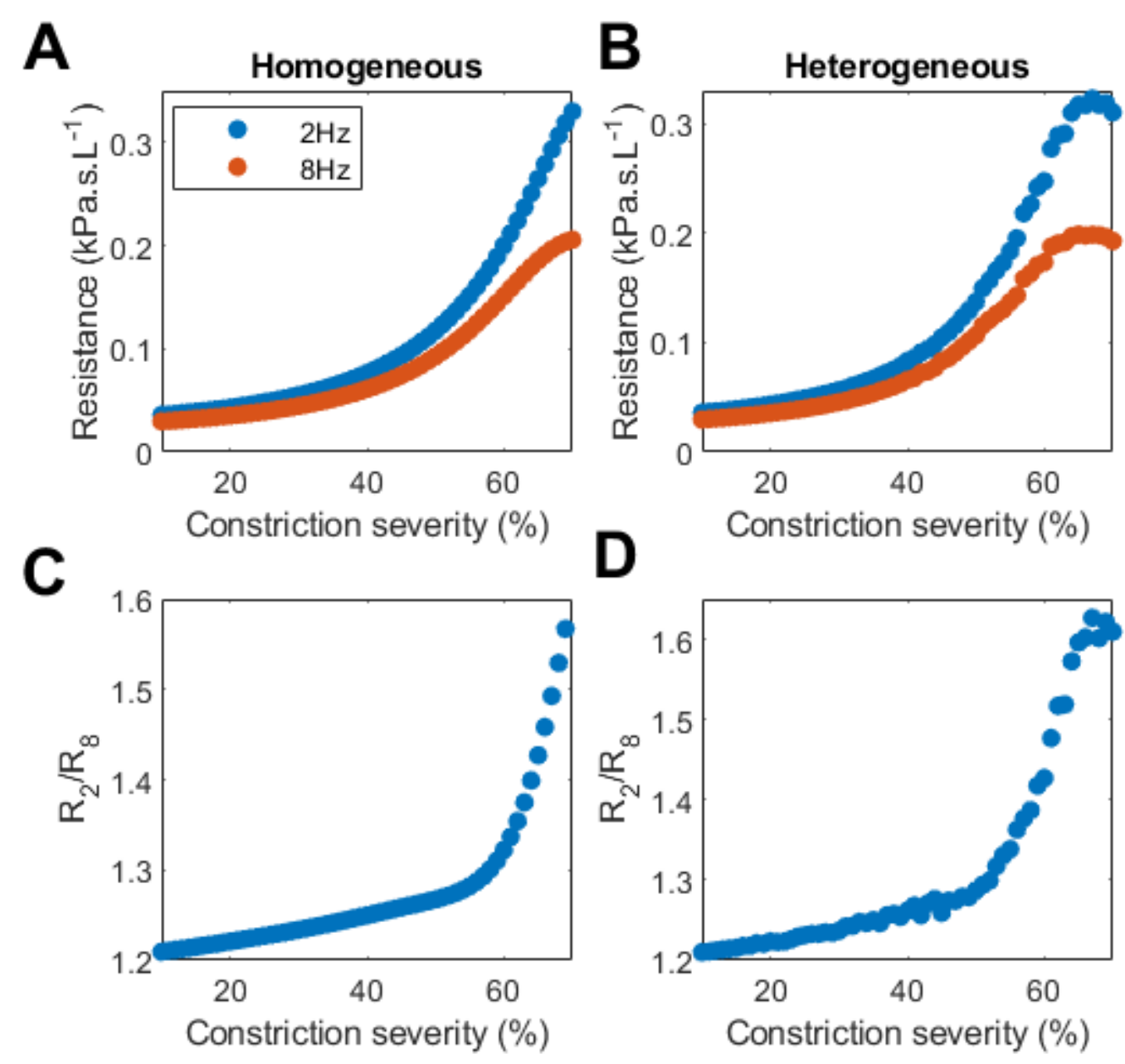

Figure 1: Simulated responses of FOT-measured resistance to small airway constriction The response of resistance at $2 \mathrm{~Hz}(\mathrm{R} 2)$ and $8 \mathrm{~Hz}(\mathrm{R} 8)$ is given for homogeneous constriction $(A, C)$ and heterogeneous constriction $(B, D)$ of the small airways. $R 2$ is seen to be consistently larger than $\mathrm{R} 8$ and increases at a faster rate in response to constriction within both schemes. This is further illustrated by the ratio R2/R8 (C, D) constantly increasing in both schemes. 
$\mathbf{A}$

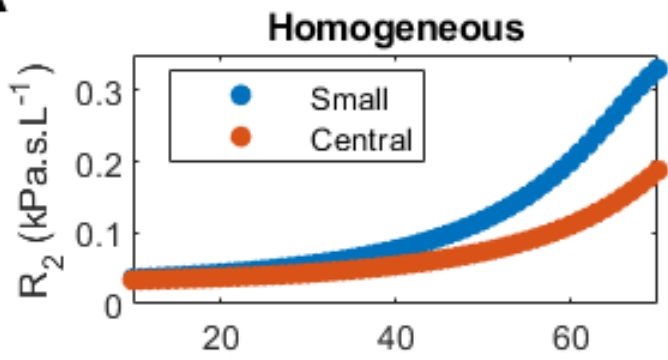

C
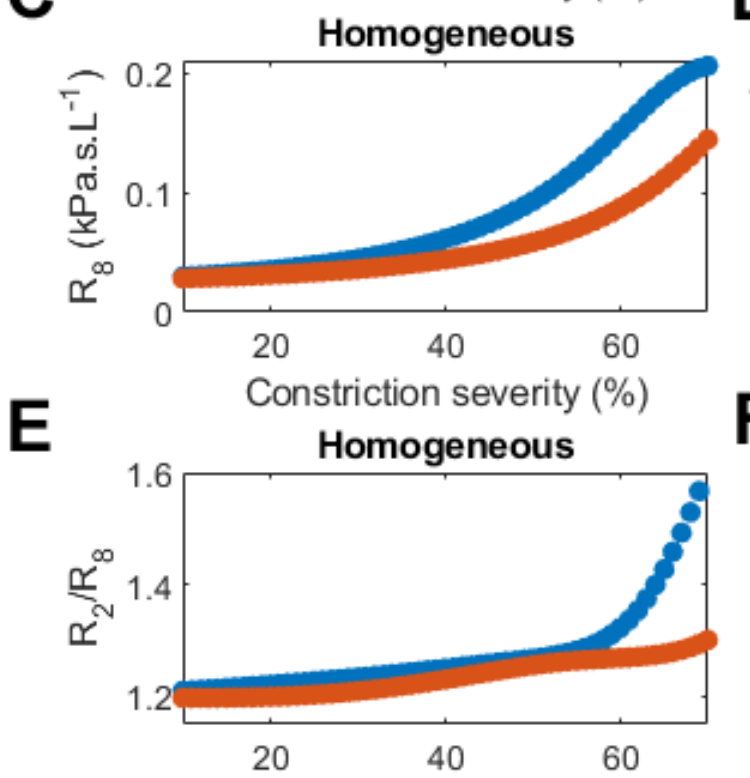

Constriction severity $(\%)$

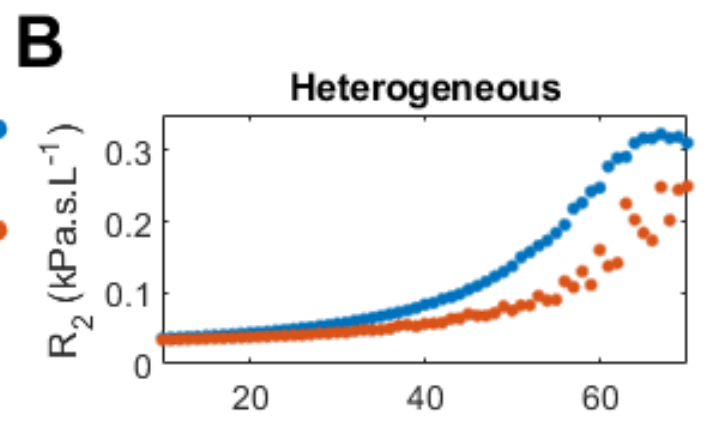

D Constriction severity (\%)
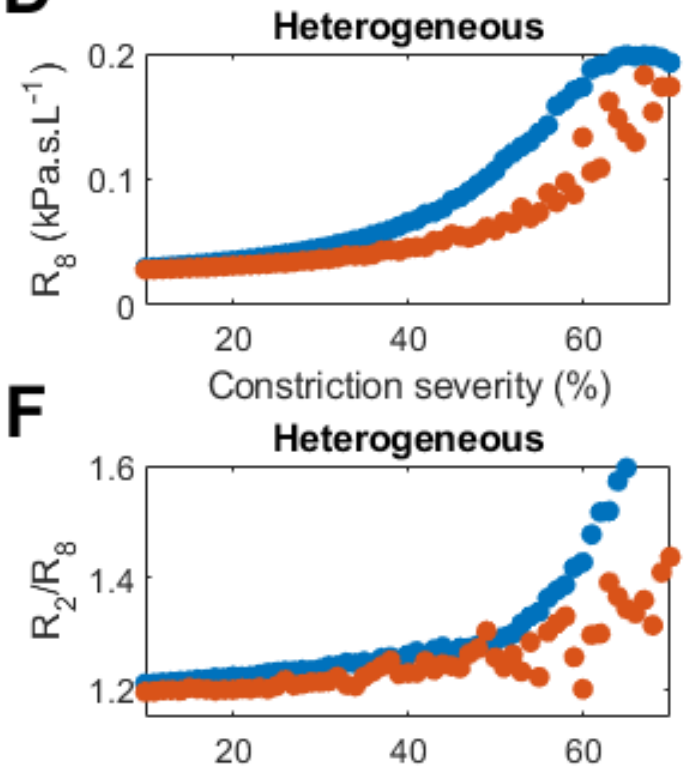

Constriction severity $(\%)$

Figure 2: Simulated response of FOT-measured resistance to small and central airway constriction. The response of $R 2(A, B)$ and $R 8(C, D)$ are given for both homogeneous $(A, C$, $E)$ and heterogeneous $(B, D, E)$ airway constriction of the small and central airways. For all cases, central airway constriction produces a smaller response than small airway constriction. However, the difference in responses between small and central airway constriction is larger for $R 2$ than $R 8$, as can be seen by considering the ratio $R 2 / R 8$ (E,F). 

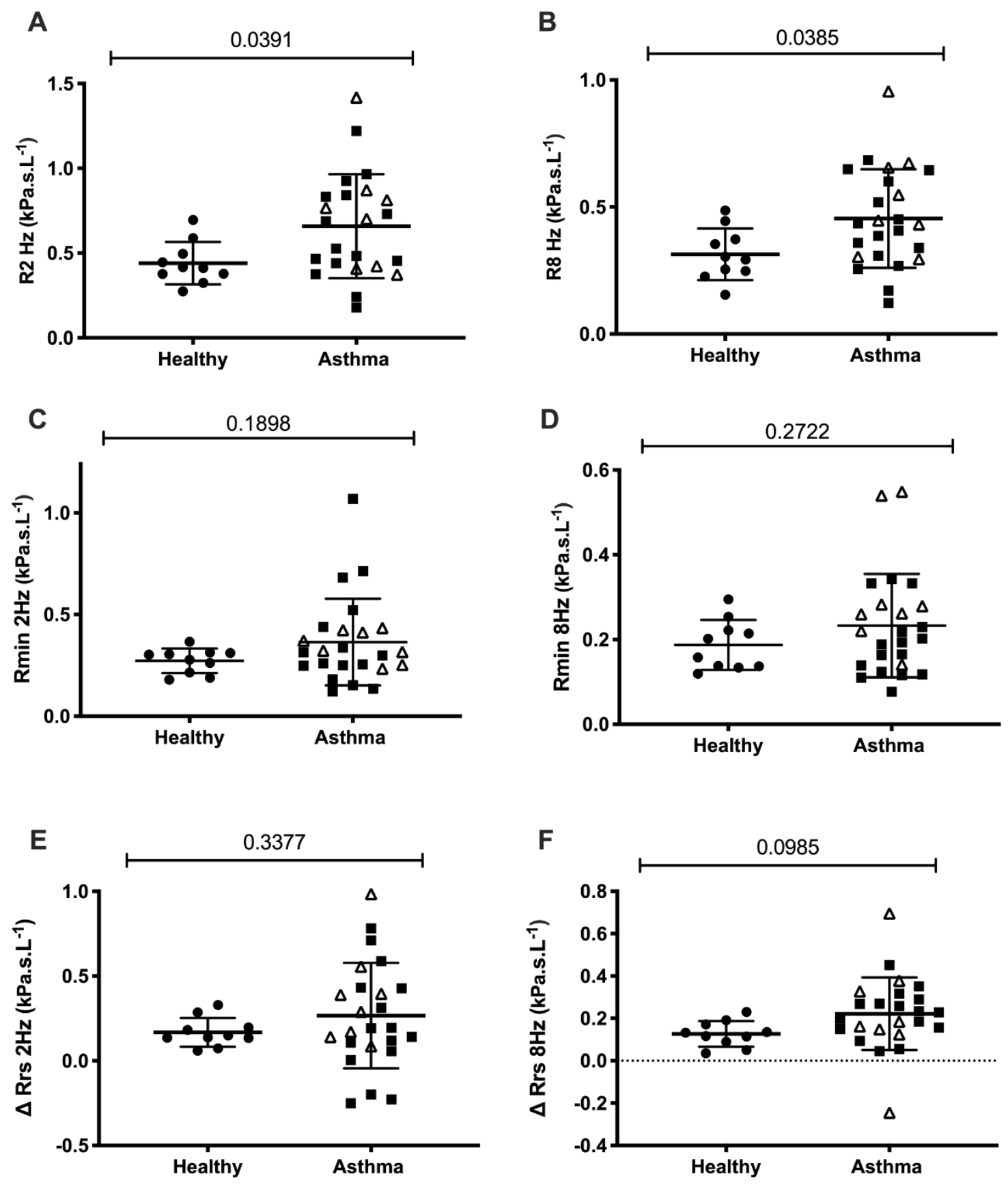

Figure 3: Mean resistance measurements in healthy and asthmatic subjects.

Measurements of baseline resistance $(A, B)$, minimum $D I$ resistance $(C, D)$ and resistive difference $(E, F)$ are given at $2 \mathrm{~Hz}(A, C, E)$ and $8 \mathrm{~Hz}(B, D, F)$. Subjects are separated based on healthy and asthmatic classification. Asthmatic subjects are sub-divided into GINA 3-4 (black squares), and GINA 5 (triangles). Median (IQR) are presented, alongside $p$-values from $t$ tests between groups. 